\title{
On a $\Phi$-Kirchhoff multivalued problem with critical growth in an Orlicz-Sobolev space
}

\author{
Giovany M. Figueiredo* \\ Universidade Federal do Pará, Faculdade de Matemática, \\ CEP: 66075-110, Belém - Pa, Brazil \\ e-mail: giovany@ufpa.br \\ Jefferson A. Santos ${ }^{\dagger}$ \\ Universidade Federal de Campina Grande, \\ Unidade Acadêmica de Matemática e Estatística, \\ CEP:58109-970, Campina Grande - PB, Brazil \\ e-mail: jefferson@dme.ufcg.edu.br
}

\begin{abstract}
This paper is concerned with the multiplicity of nontrivial solutions in an Orlicz-Sobolev space for a nonlocal problem with critical growth, involving N-functions and theory of locally Lispchitz continuous functionals. More precisely, in this paper, we study a result of multiplicity to the following multivalued elliptic problem:

$$
\left\{\begin{array}{l}
-M\left(\int_{\Omega} \Phi(|\nabla u|) d x\right) \Delta_{\Phi} u \in \partial F(., u)+\alpha h(u) \text { in } \Omega \\
u \in W_{0}^{1} L_{\Phi}(\Omega)
\end{array}\right.
$$

where $\Omega \subset \mathbb{R}^{N}$ is a bounded smooth domain, $N \geq 3, M$ is a continuous function, $\Phi$ is an $\mathrm{N}$-functions, $h$ is an odd increasing homeomorphism from $\mathbb{R}$ to $\mathbb{R}, \alpha$ is positive parameter, $\Delta_{\Phi}$ is the corresponding $\Phi$-Laplacian and $\partial F(., t)$ stands for Clarke generalized of a function $F$ linked with critical growth. We use genus theory to obtain the main result.
\end{abstract}

\section{Introduction}

Let $\Omega \subset \mathbb{R}^{N}, N \geq 3$, be a bounded domain with smooth boundary $\partial \Omega$ and consider a continuous function $\phi:(0,+\infty) \rightarrow[0,+\infty)$. The purpose of this

*Partially supported by CNPq/PQ $301242 / 2011-9$ and 200237/2012-8
†Partially supported by CNPq-Brazil grant Casadinho/Procad 552.464/2011-2 
article is to investigate the multiplicity of nontrivial solutions to the multivalued elliptic problem

$\left(P_{\alpha}\right) \quad\left\{\begin{array}{l}-M\left(\int_{\Omega} \Phi(|\nabla u|) d x\right) \Delta_{\Phi} u \in \partial F(., u)+\alpha h(u) \text { in } \Omega, \\ u \in W_{0}^{1} L_{\Phi}(\Omega),\end{array}\right.$

where $\alpha>0$ is a parameter, $\Phi$ is defined for $t \in \mathbb{R}$ by setting

$$
\Phi(t)=\int_{0}^{|t|} \phi(s) s d s
$$

$\Delta_{\Phi}$ is the operator,

$$
\Delta_{\Phi} u:=\operatorname{div}(\phi(|\nabla u|) \nabla u),
$$

$F: \Omega \times \mathbb{R} \rightarrow \mathbb{R}$ is mensurable and

$$
\partial F(x, t)=\left\{s \in \mathbb{R} ; F^{0}(x, t ; r) \geq s r, r \in \mathbb{R}\right\} .
$$

Here $F^{0}(x, t ; r)$ denotes the generalized directional derivative of $t \mapsto F(x, t)$ in direction of $r$, that is,

$$
F^{0}(x, t ; r)=\limsup _{h \rightarrow t, s \downarrow 0} \frac{F(x, h+s r)-F(x, h)}{s} .
$$

In this paper we consider

$$
F(x, t)=\int_{0}^{t} f(x, s) d s
$$

where $f: \Omega \times \mathbb{R} \rightarrow \mathbb{R}$ is mensurable and $f(x,$.$) is locally bounded in \mathbb{R}$ and

$\underline{f}(x, t)=\lim _{\epsilon \downarrow 0} \operatorname{ess} \inf \{f(x, s) ;|s-t|<\epsilon\}$ and $\bar{f}(x, t)=\lim _{\epsilon \downarrow 0} \operatorname{ess} \sup \{f(x, s) ;|s-t|<\epsilon\}$.

It is well known that

$$
\partial F(x, t)=[\underline{f}(x, t), \bar{f}(x, t)], \quad(\text { see }[14]),
$$

and that, if $f(x,$.$) is continuous then \partial F(x, t)=\{f(x, t)\}$.

Problem $\left(P_{\alpha}\right)$ with $N=3$ and $\phi(t)=4$, that is,

$$
\left\{\begin{array}{l}
-M\left(\int_{\Omega}|\nabla u|^{2} d x\right) \Delta u \in \partial F(., u)+\alpha h(u) \text { in } \Omega \\
u \in H_{0}^{1}(\Omega)
\end{array}\right.
$$

is called nonlocal because of the presence of the term $M\left(\int_{\Omega}|\nabla u|^{2} d x\right)$ which implies that the equation $(*)$ is no longer a pointwise identity. This phenomenon causes some mathematical difficulties which makes the study of such a class of 
problem particularly interesting. Besides, this class of problems has physical motivation. Indeed, the operator $M\left(\int_{\Omega}|\nabla u|^{2} d x\right) \Delta u$ appears in the Kirchhoff equation, which arises in nonlinear vibrations, namely

$$
\left\{\begin{array}{l}
u_{t t}-M\left(\int_{\Omega}|\nabla u|^{2} d x\right) \Delta u=g(x, u) \text { in } \Omega \times(0, T) \\
u=0 \text { on } \partial \Omega \times(0, T) \\
u(x, 0)=u_{0}(x), u_{t}(x, 0)=u_{1}(x)
\end{array}\right.
$$

Such a hyperbolic equation is a general version of the Kirchhoff equation

$$
\rho \frac{\partial^{2} u}{\partial t^{2}}-\left(\frac{P_{0}}{h}+\frac{E}{2 L} \int_{0}^{L}\left|\frac{\partial u}{\partial x}\right|^{2} d x\right) \frac{\partial^{2} u}{\partial x^{2}}=0
$$

presented by Kirchhoff [34. This equation extends the classical d'Alembert's wave equation by considering the effects of the changes in the length of the strings during the vibrations. The parameters in equation (1.2) have the following meanings: $L$ is the length of the string, $h$ is the area of cross-section, $E$ is the Young modulus of the material, $\rho$ is the mass density and $P_{0}$ is the initial tension.

When an elastic string with fixed ends is subjected to transverse vibrations, its length varies with the time: this introduces changes of the tension in the string. This induced Kirchhoff to propose a nonlinear correction of the classical D'Alembert equation. Later on, Woinowsky-Krieger (Nash - Modeer) incorporated this correction in the classical Euler-Bernoulli equation for the beam (plate) with hinged ends. See, for example, [6], 7] and the references therein.

This class of problems began to call attention of several researchers mainly after the work of Lions 35, where a functional analysis approach was proposed to attack it.

We have to point out that nonlocal problems also appear in other fields as, for example, biological systems where $u$ describes a process which depends on the average of itself (for example, population density). See, for example, [2] and its references.

The reader may consult [2], 3], 28, 36] and the references therein, for more information on nonlocal problems.

On the other hand, in this study, the nonlinearity $f$ can be discontinuous. The interest in the study of nonlinear partial differential equations with discontinuous nonlinearities has increased because many free boundary problems arising in mathematical physics may be stated in this form. Among these problems, we have the obstacle problem, the seepage surface problem, and the Elenbaas equation, see for example [14, [15] and [16].

For enunciate the main result, we need to give some hypotheses on the functions $M, \phi, h$ and $f$.

The hypotheses on the function $\phi:(0,+\infty) \rightarrow(0,+\infty)$ of $C^{1}$ class are the following: 
$\left(\phi_{1}\right)$ For all $t>0$,

$$
\phi(t)>0 \text { and }(\phi(t) t)^{\prime}>0 .
$$

$\left(\phi_{2}\right)$ There exist $l \in\left(\frac{N}{2}, N\right), l<m<\frac{l^{*}}{2}=\frac{l N}{2(N-l)}$ such that

$$
l \leq \frac{\phi(t) t^{2}}{\Phi(t)} \leq m
$$

for $t>0$, where $\Phi(t)=\int_{0}^{|t|} \phi(s) s d s$.

Through this paper, we assume that $h$ is an odd increasing homeomorphism from $\mathbb{R}$ to $\mathbb{R}$, verifying

$\left(H_{0}\right)$ There exist $1<h_{0}<h_{1}<l$ such that

$$
h_{0} \leq \frac{h(t) t}{H(t)} \leq h_{1}, t>0
$$

where $H(t)=\int_{0}^{t} h(s) d s$.

Considering $\widetilde{H}(t):=\int_{0}^{t} h^{-1}(s) d s$, then we can obtain complementary N-function which define corresponding Orlicz space $L_{H}(\Omega)$.

The hypothesis on the continuous function $M: \mathbb{R}^{+} \rightarrow \mathbb{R}^{+}$is the following:

$\left(M_{1}\right)$ There exists $\sigma>0$ such that

$$
M(t) \geq \sigma \text { for all } t \geq 0 .
$$

$\left(M_{2}\right)$ There exists $\theta \in\left(2 m, l^{*}\right)$ such that

$$
\widehat{M}(t)-\frac{m}{\theta} M(t) t \geq 0, \text { for all } t \geq 0,
$$

where $\widehat{M}(t)=\int_{0}^{t} M(s) d s$.

The hypotheses on the function $f: \Omega \times \mathbb{R} \rightarrow \mathbb{R}$ are the following:

$\left(f_{1}\right) f(x, t)$ is odd with respect $t$, there exist $b_{0}, b_{1}>0$ and $a_{0} \geq 0$ such that

$$
b_{0} \phi_{*}(t) t \leq f(x, t) \leq b_{1} \phi_{*}(t) t, t \geq a_{0} \text { and } x \in \Omega,
$$

with $1<\frac{b_{1}}{b_{0}}<\frac{l^{*}}{\theta}$, where $\phi_{*}(t) t$ is such that Sobolev conjugate function $\Phi_{*}$ of $\Phi$ is its primitive, that is, $\Phi_{*}(t)=\int_{0}^{|t|} \phi_{*}(s) s d s$.

$\left(f_{2}\right)$ Moreover, if $a_{0}>0$, we consider $f(x, t)=0$ for all $|t|<a_{0}$ and $x \in \Omega$.

The main result of this paper is: 
Theorem 1.1 Assume that conditions $\left(\phi_{1}\right),\left(\phi_{2}\right),\left(H_{0}\right),\left(M_{1}\right),\left(M_{2}\right),\left(f_{1}\right),\left(f_{2}\right)$ hold. If $f$ and $\bar{f}$ are $N$-functions, then for $\alpha=\alpha\left(\theta, h_{0}, b_{0}, b_{1}, l, m, \sigma, S_{N}\right)>0$, $\left.a_{0}=a_{0} \overline{(H}(1), \sigma, l,|\Omega|, \alpha, h_{1}\right)>0$ sufficiently small (or $a_{0}=0$ ), the problem $\left(P_{\alpha}\right)$ has infinitely many solutions.

An Application: Consider the equation

$\left(A_{1}\right)\left\{\begin{array}{l}-\left(a \int_{\Omega} \Phi(|\nabla u|) d x+b\right) \Delta_{\Phi} u=\widehat{H}\left(|u|-a_{0}\right) \phi_{*}(|u|) u+\alpha h(u) \text { in } \Omega, \\ u \in W_{0}^{1} L_{\Phi}(\Omega),\end{array}\right.$

where $a, b, a_{0}>0$ and $\widehat{H}$ is the Heaviside function. We claim that $\left(A_{1}\right)$ admits infinitely many solutions in $W_{0}^{1, \Phi}(\Omega)$, for $\alpha, a_{0}>0$ sufficiently small. Indeed, let $f(x, t)=\widehat{H}\left(|t|-a_{0}\right) \phi_{*}(|t|) t$ and set

$$
F(x, u)=\int_{0}^{u} f(x, t) d t=\chi_{\left[|u| \geq a_{0}\right]}\left(\Phi_{*}(u)-\Phi_{*}\left(a_{0}\right)\right) .
$$

Next consider the associated multivalued equation

$$
\left\{-\left(a \int_{\Omega} \Phi(|\nabla u|) d x+b\right) \Delta_{\Phi} u \in \partial F(., u)+\alpha h(u) \text { in } \Omega .\right.
$$

We will show that the assumptions of Theorem 1.1 hold true. Indeed, since $\Omega$ is bounded, it is straightforward to check that conditions $\left(M_{1}\right),\left(M_{2}\right),\left(f_{1}\right)$ and $\left(f_{2}\right)$ hold. Thus by Theorem 1.1. $\left(A_{2}\right)$ admits infinitely many solutions in $W_{0}^{1, \Phi}(\Omega)$ with $\alpha, a_{0}>0$ sufficiently small. Finally, since that $\left|\left[|u|=a_{0}\right]\right|=0$ the problem $\left(A_{1}\right)$ has infinitely many solutions.

In the last twenty years the study on nonlocal problems of the type

$$
\left\{\begin{array}{l}
-M\left(\int_{\Omega}|\nabla u|^{2} d x\right) \Delta u=g(x, u) \text { in } \Omega, \\
u \in H_{0}^{1}(\Omega)
\end{array}\right.
$$

grew exponentially. That was, probably, by the difficulties existing in this class of problems and that do not appear in the study of local problems, as well as due to their significance in applications. Without hope of being thorough, we mention some articles with multiplicity results and that are related with our main result. We will restrict our comments to the works that have emerged in the last four years

The problem $(K)$ was studied in 28 . The version with p-Laplacian operator was studied in 22]. In both cases, the authors showed a multiplicity result using genus theory. In 32 and 37 the authors showed a multiplicity result for the problem $(K)$ using the Fountain theorem and the Symmetric Mountain Pass theorem. In all these articles the nonlinearity is continuous. The case discontinuous was studied in 23. With a nonlinearity of the Heaviside type the authors showed a existence of two solutions via Mountain Pass Theorem and Ekeland's Variational Principle. 
In [18 the author showed the existence of two solutions for a problem involving $\Phi$-Kirchhoff operator and nonlinearity of variable exponent type and subcritical growth. He used the mountain pass theorem combined with the minimum principle.

In this work we extend the studies found in the papers above in the following sense:

a) We cannot use the classical Clark's Theorem for $C^{1}$ functional (see [24, Theorem 3.6]), because in our case, the energy functional is only locally Lipschitz continuous. Thus, in all section 5 we adapt for nondifferentiable functionals an argument found in $[$.

b) Unlike [18 and 23, we show a result of multiplicity using genus theory considering a nonlinearity that can have a number enumerable of discontinuities.

c) Problem $\left(P_{\alpha}\right)$ possesses more complicated nonlinearities, for example:

(i) $\Phi(t)=t^{p_{0}}+t^{p_{1}}, 1<p_{0}<p_{1}<N$ and $p_{1} \in\left(p_{0}, p_{0}^{*}\right)$.

(ii) $\Phi(t)=\left(1+t^{2}\right)^{\gamma}-1, \gamma \in\left(1, \frac{N}{N-2}\right)$.

(iii) $\Phi(t)=t^{p} \log (1+t)$ with $1<p_{0}<p<N-1$, where $p_{0}=\frac{-1+\sqrt{1+4 N}}{2}$.

(iv) $\Phi(t)=\int_{0}^{t} s^{1-\theta}\left(\sinh ^{-1} s\right)^{\beta} d s, 0 \leq \theta \leq 1, \beta>0$.

Before concluding this introduction, it is very important to say that in the literature, we find many papers where the authors study the existence and multiplicity of solution for problems involving the $\Phi$-Laplacian operator, see, for example, [10, 13], 17, 26], 38, and references therein.

The paper is organized as follows. In the next section we present a brief review on Orlicz-Sobolev spaces. In section 3 we recall some definitions and basic results on the critical point theory of locally Lipschitz continuous functionals. In Section 4 we present just some preliminary results involving genus theory that will be used in this work. In the Section 5 we prove Theorem 1.1

\section{A brief review on Orlicz-Sobolev spaces}

Let $\varphi$ be a real-valued function defined in $[0, \infty)$ and having the following properties:

a) $\varphi(0)=0, \varphi(t)>0$ if $t>0$ and $\lim _{t \rightarrow \infty} \varphi(t)=\infty$.

b) $\varphi$ is nondecreasing, that is, $s>t$ implies $\varphi(s) \geq \varphi(t)$.

c) $\varphi$ is right continuous, that is, $\lim _{s \rightarrow t^{+}} \varphi(s)=\varphi(t)$.

Then, the real-valued function $\Phi$ defined on $\mathbb{R}$ by

$$
\Phi(t)=\int_{0}^{|t|} \varphi(s) d s
$$

is called an N-function. For an N-function $\Phi$ and an open set $\Omega \subseteq \mathbb{R}^{N}$, the Orlicz space $L_{\Phi}(\Omega)$ is defined (see [1]). When $\Phi$ satisfies $\Delta_{2}$-condition, that is, 
when there are $t_{0} \geq 0$ and $K>0$ such that $\Phi(2 t) \leq K \Phi(t)$, for all $t \geq t_{0}$, the space $L_{\Phi}(\Omega)$ is the vectorial space of the measurable functions $u: \Omega \rightarrow \mathbb{R}$ such that

$$
\int_{\Omega} \Phi(|u|) d x<\infty
$$

The space $L_{\Phi}(\Omega)$ endowed with Luxemburg norm, that is, the norm given by

$$
|u|_{\Phi}=\inf \left\{\tau>0: \int_{\Omega} \Phi\left(\frac{|u|}{\tau}\right) d x \leq 1\right\},
$$

is a Banach space. The complement function of $\Phi$, denoted by $\widetilde{\Phi}$, is given by the Legendre transformation, that is

$$
\widetilde{\Phi}(s)=\max _{t \geq 0}\{s t-\Phi(t)\} \text { for } s \geq 0 .
$$

These $\Phi$ and $\widetilde{\Phi}$ are complementary each other. Involving the functions $\Phi$ and $\widetilde{\Phi}$, we have the Young's inequality given by

$$
s t \leq \Phi(t)+\widetilde{\Phi}(s) .
$$

Using the above inequality, it is possible to prove the following Hölder type inequality

$$
\left|\int_{\Omega} u v d x\right| \leq 2|u|_{\Phi}|v|_{\widetilde{\Phi}} \forall u \in L_{\Phi}(\Omega) \text { and } v \in L_{\widetilde{\Phi}}(\Omega) .
$$

Hereafter, we denote by $W_{0}^{1} L_{\Phi}(\Omega)$ the Orlicz-Sobolev space obtained by the completion of $C_{0}^{\infty}(\Omega)$ with norm

$$
\|u\|_{\Phi}=|u|_{\Phi}+|\nabla u|_{\Phi} .
$$

When $\Omega$ is bounded, there is $c>0$ such that

$$
|u|_{\Phi} \leq c|\nabla u|_{\Phi} .
$$

In this case, we can consider

$$
\|u\|_{\Phi}=|\nabla u|_{\Phi} .
$$

Another important function related to function $\Phi$, is the Sobolev conjugate function $\Phi_{*}$ of $\Phi$ defined by

$$
\Phi_{*}^{-1}(t)=\int_{0}^{t} \frac{\Phi^{-1}(s)}{s^{(N+1) / N}} d s, t>0 .
$$

Another important inequality was proved by Donaldson and Trudinger [27, which establishes that for all open $\Omega \subset \mathbb{R}^{N}$ and there is a constant $S_{N}=S(N)>0$ such that

$$
|u|_{\Phi_{*}} \leq S_{N}|\nabla u|_{\Phi}, u \in W_{0}^{1, \Phi}(\Omega) .
$$


This inequality shows the below embedding is continuous

$$
W_{0}^{1, \Phi}(\Omega) \underset{\text { cont }}{\hookrightarrow} L_{\Phi_{*}}(\Omega) .
$$

If bounded domain $\Omega$ and the limits below hold

$$
\limsup _{t \rightarrow 0} \frac{B(t)}{\Phi(t)}<+\infty \text { and } \limsup _{|t| \rightarrow+\infty} \frac{B(t)}{\Phi_{*}(t)}=0,
$$

the embedding

$$
W_{0}^{1, \Phi}(\Omega) \hookrightarrow L_{B}(\Omega)
$$

is compact.

The hypotheses $\left(\phi_{1}\right)-\left(\phi_{2}\right)$ implies that $\Phi, \widetilde{\Phi}, \Phi_{*}$ and $\widetilde{\Phi}_{*}$ satisfy $\Delta_{2}$ condition. This condition allows us conclude that:

1) $u_{n} \rightarrow 0$ in $L_{\Phi}(\Omega)$ if, and only if, $\int_{\Omega} \Phi\left(u_{n}\right) d x \rightarrow 0$.

2) $L_{\Phi}(\Omega)$ is separable and ${\overline{C_{0}^{\infty}(\Omega)}}^{|\cdot|_{\Phi}}=L_{\Phi}(\Omega)$.

3) $L_{\Phi}(\Omega)$ is reflexive and its dual is $L_{\widetilde{\Phi}}(\Omega)$ (see [1]).

Under assumptions $\left(\phi_{1}\right)-\left(\phi_{2}\right)$, some elementary inequalities listed in the following lemmas are valid. For the proofs, see [29].

Lemma 2.1 Assume $\left(\phi_{1}\right)-\left(\phi_{2}\right)$. Then,

$$
\Phi(t)=\int_{0}^{|t|} s \phi(s) d s
$$

is a $N$-function with $\Phi, \widetilde{\Phi} \in \Delta_{2}$. Hence, $L_{\Phi}(\Omega), W^{1, \Phi}(\Omega)$ and $W_{0}^{1, \Phi}(\Omega)$ are reflexive and separable spaces.

Lemma 2.2 The functions $\Phi, \Phi_{*}, \widetilde{\Phi}$ and $\widetilde{\Phi}_{*}$ satisfy the inequality

$$
\widetilde{\Phi}(\phi(t) t) \leq \Phi(2 t) \text { and } \widetilde{\Phi}_{*}\left(\phi_{*}(t) t\right) \leq \Phi_{*}(2 t) \forall t \geq 0 .
$$

Lemma 2.3 Assume that $\left(\phi_{1}\right)-\left(\phi_{2}\right)$ hold and let $\xi_{0}(t)=\min \left\{t^{l}, t^{m}\right\}$, $\xi_{1}(t)=\max \left\{t^{l}, t^{m}\right\}$, for all $t \geq 0$. Then,

$$
\xi_{0}(\rho) \Phi(t) \leq \Phi(\rho t) \leq \xi_{1}(\rho) \Phi(t) \quad \text { for } \rho, t \geq 0
$$

and

$$
\xi_{0}\left(|u|_{\Phi}\right) \leq \int_{\Omega} \Phi(u) \leq \xi_{1}\left(|u|_{\Phi}\right) \quad \text { for } u \in L_{\Phi}(\Omega) .
$$

Lemma 2.4 The function $\Phi_{*}$ satisfies the following inequality

$$
l^{*} \leq \frac{\Phi_{*}^{\prime}(t) t}{\Phi_{*}(t)} \leq m^{*} \text { for } t>0 .
$$

As an immediate consequence of the Lemma 2.4, we have the following result 
Lemma 2.5 Assume that $\left(\phi_{1}\right)-\left(\phi_{2}\right)$ hold and let $\xi_{2}(t)=\min \left\{t^{l^{*}}, t^{m^{*}}\right\}$, $\xi_{3}(t)=\max \left\{t^{l^{*}}, t^{m^{*}}\right\}$ for all $t \geq 0$. Then,

$$
\xi_{2}(\rho) \Phi_{*}(t) \leq \Phi_{*}(\rho t) \leq \xi_{3}(\rho) \Phi_{*}(t) \text { for } \rho, t \geq 0
$$

and

$$
\xi_{2}\left(|u|_{\Phi_{*}}\right) \leq \int_{\Omega} \Phi_{*}(u) d x \leq \xi_{3}\left(|u|_{\Phi_{*}}\right) \quad \text { for } u \in L_{A_{*}}(\Omega) .
$$

Lemma 2.6 Let $\widetilde{\Phi}$ be the complement of $\Phi$ and put

$$
\xi_{4}(s)=\min \left\{s^{\frac{l}{l-1}}, s^{\frac{m}{m-1}}\right\} \text { and } \xi_{5}(s)=\max \left\{s^{\frac{l}{l-1}}, s^{\frac{m}{m-1}}\right\}, s \geq 0 .
$$

Then the following inequalities hold

$$
\xi_{4}(r) \widetilde{\Phi}(s) \leq \widetilde{\Phi}(r s) \leq \xi_{5}(r) \widetilde{\Phi}(s), r, s \geq 0
$$

and

$$
\xi_{4}\left(|u|_{\widetilde{\Phi}}\right) \leq \int_{\Omega} \widetilde{\Phi}(u) d x \leq \xi_{5}\left(|u|_{\widetilde{\Phi}}\right), u \in L_{\widetilde{\Phi}}(\Omega) .
$$

The next result is a version of Brezis-Lieb's Lemma [11 for Orlicz-Sobolev spaces and the proof can be found in 31.

Lemma 2.7 Let $\Omega \subset \mathbb{R}^{N}$ open set and $\Phi: \mathbb{R} \rightarrow[0, \infty)$ an $N$-function satisfies $\Delta_{2}-$ condition. If the complementary function $\widetilde{\Phi}$ satisfies $\Delta_{2}-$ condition, $\left(f_{n}\right)$ is bounded in $L_{\Phi}(\Omega)$, such that

$$
f_{n}(x) \rightarrow f(x) \text { a.s } x \in \Omega,
$$

then

$$
f_{n} \rightarrow f \text { in } L_{\Phi}(\Omega)
$$

\section{Technical results on locally Lipschitz function and variational framework}

In this section, for the reader's convenience, we recall some definitions and basic results on the critical point theory of locally Lipschitz continuous functionals as developed by Chang [14, Clarke [20, 21] and Grossinho \& Tersin [30.

Let $X$ be a real Banach space. A functional $J: X \rightarrow \mathbb{R}$ is locally Lipschitz continuous, $J \in \operatorname{Lip}_{\text {loc }}(X, \mathbb{R})$ for short, if given $u \in X$ there is an open neighborhood $V:=V_{u} \subset X$ and some constant $K=K_{V}>0$ such that

$$
\left|J\left(v_{2}\right)-J\left(v_{1}\right)\right| \leq K\left\|v_{2}-v_{1}\right\|, v_{i} \in V, i=1,2 .
$$

The directional derivative of $J$ at $u$ in the direction of $v \in X$ is defined by 


$$
J^{0}(u ; v)=\limsup _{h \rightarrow 0, \sigma \downarrow 0} \frac{J(u+h+\sigma v)-I(u+h)}{\sigma} .
$$

The generalized gradient of $J$ at $u$ is the set

$$
\partial J(u)=\left\{\mu \in X^{*} ;\langle\mu, v\rangle \leq J^{0}(u ; v), v \in X\right\} .
$$

Since $J^{0}(u ; 0)=0, \partial J(u)$ is the subdifferential of $J^{0}(u ; 0)$. Moreover, $J^{0}(u ; v)$ is the support function of $\partial J(u)$ because

$$
J^{0}(u ; v)=\max \{\langle\xi, v\rangle ; \xi \in \partial J(u)\} .
$$

The generalized gradient $\partial J(u) \subset X^{*}$ is convex, non-empty and weak*compact, and

$$
m_{J}(u)=\min \left\{\|\mu\|_{X^{*}} ; \mu \in \partial J(u)\right\} .
$$

Moreover,

$$
\partial J(u)=\left\{J^{\prime}(u)\right\}, \text { if } J \in C^{1}(X, \mathbb{R}) .
$$

A critical point of $J$ is an element $u_{0} \in X$ such that $0 \in \partial J\left(u_{0}\right)$ and a critical value of $J$ is a real number $c$ such that $J\left(u_{0}\right)=c$ for some critical point $u_{0} \in X$.

About variational framework, we say that $u \in W_{0}^{1} L_{\Phi}(\Omega)$ is a weak solution of the problem $\left(P_{\alpha}\right)$ if it verifies the hemivariational inequality

$M\left(\int_{\Omega} \Phi(|\nabla u|) d x\right) \int_{\Omega} \phi(|\nabla u|) \nabla u \nabla v d x-\int_{\Omega} F^{0}(x, u ; v) d x-\alpha \int_{\Omega} h(u) v d x \leq 0$

for all $v \in W_{0}^{1} L_{\Phi}(\Omega)$ and moreover the set $\left\{x \in \Omega ;|u| \geq a_{0}\right\}$ has positive measure. Thus, weak solutions of $\left(P_{\alpha}\right)$ are critical points of the functional

$$
J_{\alpha}(u)=\widehat{M}\left(\int_{\Omega} \Phi(|\nabla u|) d x\right)-\int_{\Omega} F(x, u) d x-\alpha \int_{\Omega} H(u) d x,
$$

where $\widehat{M}(t)=\int_{0}^{t} M(s) d s$. In order to use variational methods, we first derive some results related to the Palais-Smale compactness condition for the problem $\left(P_{\alpha}\right)$.

We say that a sequence $\left(u_{n}\right) \subset W_{0}^{1} L_{\Phi}(\Omega)$ is a Palais-Smale sequence for the locally lipschitz functional $J_{\alpha}$ associated of problem $\left(P_{\alpha}\right)$ if

$$
J_{\alpha}\left(u_{n}\right) \rightarrow c_{\alpha} \text { and } m_{J_{\alpha}}\left(u_{n}\right) \rightarrow 0 \text { in }\left(W_{0}^{1} L_{\Phi}(\Omega)\right)^{*} .
$$

If (3.1) implies the existence of a subsequence $\left(u_{n_{j}}\right) \subset\left(u_{n}\right)$ which converges in $W_{0}^{1, \Phi}(\Omega)$, we say that these one functionals satisfies the nonsmooth $(P S)_{c_{\alpha}}$ condition. 
Note that $J_{\alpha} \in \operatorname{Lip}_{l o c}\left(W_{0}^{1, \Phi}(\Omega), \mathbb{R}\right)$ and from convex analysis theory, for all $w \in \partial J_{\alpha}(u)$,

$$
\langle w, v\rangle=M\left(\int_{\Omega} \Phi(|\nabla u|) d x\right) \int_{\Omega} \phi(|\nabla u|) \nabla u \nabla v d x-\langle\rho, v\rangle-\alpha \int_{\Omega} h(u) v d x
$$

for some $\rho \in \partial J_{F}(u)$, where

$$
J_{F}(u)=\int_{\Omega} F(x, u) d x, u \in W_{0}^{1, \Phi}(\Omega) .
$$

We have $J_{F} \in \operatorname{Lip}_{\text {loc }}\left(L_{\Phi_{*}}(\Omega)\right), \partial J_{F}(u) \subset L_{\widetilde{\Phi}_{*}}(\Omega)$.

The next lemma is similar to [4, theorem 4.1] and its proof will be omitted.

Lemma 3.1 Assume that $\underline{f}, \bar{f}$ are $N$-measurable and that $\left(\phi_{1}\right)-\left(\phi_{2}\right)$ and $\left(f_{1}\right)$ hold. Then

(i) $\partial J_{F}(u) \subset \partial F(x, u)$, a.e. $x \in \Omega, u \in L_{\Phi_{*}}(\Omega)$.

(ii) $\left.\partial J_{F}\right|_{W_{0}^{1, \Phi}}(\Omega)(u)=\partial J_{F}(u), u \in W_{0}^{1, \Phi}(\Omega)$.

\section{Results involving genus}

We will start by considering some basic notions on the Krasnoselskii genus that we will use in the proof of our main results.

Let $E$ be a real Banach space. Let us denote by $\mathfrak{A}$ the class of all closed subsets $A \subset E \backslash\{0\}$ that are symmetric with respect to the origin, that is, $u \in A$ implies $-u \in A$.

Definition 4.1 Let $A \in \mathfrak{A}$. The Krasnoselskii genus $\gamma(A)$ of $A$ is defined as being the least positive integer $k$ such that there is an odd mapping $\phi \in C\left(A, \mathbb{R}^{k}\right)$ such that $\phi(x) \neq 0$ for all $x \in A$. If $k$ does not exist we set $\gamma(A)=\infty$. Furthermore, by definition, $\gamma(\emptyset)=0$.

In the sequel we will establish only the properties of the genus that will be used through this work. More information on this subject may be found in the references by [5, [12, 19], 24] and [33].

Proposition 4.1 Let $E=\mathbb{R}^{N}$ and $\partial \Omega$ be the boundary of an open, symmetric and bounded subset $\Omega \subset \mathbb{R}^{N}$ with $0 \in \Omega$. Then $\gamma(\partial \Omega)=N$.

Corollary $4.1 \gamma\left(\mathcal{S}^{N-1}\right)=N$ where $\mathcal{S}^{N-1}$ is a unit sphere of $\mathbb{R}^{N}$.

Proposition 4.2 If $K \in \mathfrak{A}, 0 \notin K$ and $\gamma(K) \geq 2$, then $K$ has infinitely many points. 


\section{Proof of Theorem 1.1}

The plan of the proof is to show that the set of critical points of the functional $J_{\alpha}$ is compact, symmetric, does not contain the zero and has genus more than 2. Thus, our main result is a consequence of Proposition 4.2

Using $\left(M_{1}\right)$ and Orlicz's embedding, we get

$$
\begin{aligned}
J_{\alpha}(u) \geq & \sigma \int_{\Omega} \Phi(|\nabla u|) d x-c_{1} \alpha \eta_{1} \circ \xi_{0}^{-1}\left(\int_{\Omega} \Phi(|\nabla u|) d x\right) \\
& -c_{2} \xi_{3} \circ \xi_{0}^{-1}\left(\int_{\Omega} \Phi(|\nabla u|) d x\right):=g\left(\int_{\Omega} \Phi(|\nabla u|) d x\right),
\end{aligned}
$$

where

$$
g(t)=\left\{\begin{array}{l}
\sigma t-c_{1} \alpha t^{\frac{h_{0}}{m}}-c_{2} t^{\frac{l^{*}}{m}}, \text { se } t \in(0,1] \\
\sigma t-c_{1} \alpha t^{\frac{h_{1}}{l}}-c_{2} t^{\frac{m^{*}}{l}}, \text { se } t \in[1,+\infty) .
\end{array}\right.
$$

Thus, there exists $\alpha_{\#}>0$ such that, if $\alpha \in\left(0, \alpha_{\#}\right)$, then $g$ attains its positive maximum. Let us assume $\alpha \in\left(0, \alpha_{\#}\right)$, choosing $A_{\alpha}$ and $B_{\alpha}$ as the first and second root of $g$, we make the following of the truncation $J_{\alpha}$. Fix $\Psi \in C_{0}^{\infty}([0,+\infty)), 0 \leq \Psi(t) \leq 1$ for all $t \in[0,+\infty)$, such that $\Psi(t)=1$ if $t \in\left[0, A_{\alpha}\right]$ and $\Psi(t)=0$ if $t \in\left[B_{\alpha},+\infty\right)$. Now, we consider the truncated functional

$$
I_{\alpha}(u)=\widehat{M}\left(\int_{\Omega} \Phi(|\nabla u|) d x\right)-\alpha \int_{\Omega} H(u) d x-\Psi\left(\int_{\Omega} \Phi(|\nabla u|) d x\right) \int_{\Omega} F(x, u) d x .
$$

From $\left(M_{1}\right)$ and Orlicz's embedding, we have that

$$
\begin{aligned}
I_{\alpha}(u) \geq & \sigma \int_{\Omega} \Phi(|\nabla u|) d x-\alpha \widetilde{c}_{1} \eta_{1} \circ \xi_{0}^{-1}\left(\int_{\Omega} \Phi(|\nabla u|) d x\right) \\
& -\widetilde{c}_{2} \Psi\left(\int_{\Omega} \Phi(|\nabla u|) d x\right) \xi_{3} \circ \xi_{0}^{-1}\left(\int_{\Omega} \Phi(|\nabla u|) d x\right) \\
:= & \bar{g}\left(\int_{\Omega} \Phi(|\nabla u|) d x\right),
\end{aligned}
$$

where

$$
\bar{g}(t)=\left\{\begin{array}{l}
\sigma t-\widetilde{c}_{1} \alpha t^{\frac{h_{0}}{m}}-\widetilde{c}_{2} \Psi(t) t^{\frac{l^{*}}{m}}, \text { se } t \in(0,1] \\
\sigma t-\widetilde{c}_{1} \alpha t^{\frac{h_{1}}{l}}-\widetilde{c}_{2} \Psi(t) t^{\frac{m^{*}}{l}}, \text { se } t \in[1,+\infty)
\end{array}\right.
$$

Now, we will show that $I_{\alpha}$ satisfy the local Palais-Smale condition. For this, we need the following technical result involving the functional $J_{\alpha}$.

Lemma 5.1 Let $\left(u_{n}\right) \subset W_{0}^{1, \Phi}(\Omega)$ be a bounded sequence such that

$$
J_{\alpha}\left(u_{n}\right) \rightarrow c_{\alpha} \text { and } m^{J_{\alpha}}\left(u_{n}\right) \rightarrow 0 .
$$

There exists $\alpha>0$ small enough such that, if

$c_{\alpha}<\omega_{N}:=\frac{1}{2} \min \left\{\left(\frac{l \sigma}{b_{1} m^{*}}\right)^{\frac{\beta}{\beta-1}} S_{N}^{-\frac{\alpha}{\beta-1}} ; \alpha \in\left\{l^{*}, m^{*}\right\}\right.$ and $\left.\beta \in\left\{\frac{l^{*}}{l}, \frac{m^{*}}{l}, \frac{l^{*}}{m}, \frac{m^{*}}{m}\right\}\right\}$, 
then up a subsequence, $\left(u_{n}\right)$ is strongly in $L_{\Phi_{*}}(\Omega)$.

Proof: Since $\left(u_{n}\right)$ is bounded in $W_{0}^{1} L_{\Phi}(\Omega)$, we can extract a subsequence of $\left(u_{n}\right)$, still denoted by $\left(u_{n}\right)$ and $u \in W_{0}^{1, \Phi}(\Omega)$, such that

- $u_{n} \rightarrow u$ in $W_{0}^{1, \Phi}(\Omega)$;

- $u_{n} \rightarrow u$ in $L_{\Phi_{*}}(\Omega)$;

- $u_{n} \rightarrow u$ in $L_{\Phi}(\Omega)$;

- $u_{n}(x) \rightarrow u(x)$ a.e. in $\Omega$;

From the concentration compactness lemma of Lions in Orlicz-Sobolev space found in [29], there exist two nonnegative measures $\mu, \nu \in \mathcal{M}(\Omega)$, a countable set $\mathcal{J}$, points $\left\{x_{j}\right\}_{j \in \mathcal{J}}$ in $\Omega$ and sequences $\left\{\mu_{j}\right\}_{j \in \mathcal{J}},\left\{\nu_{j}\right\}_{j \in \mathcal{J}} \subset[0,+\infty)$, such that

$$
\begin{array}{r}
\Phi\left(\left|\nabla u_{n}\right|\right) \rightarrow \mu \geq \Phi(|\nabla u|)+\sum_{j \in \mathcal{J}} \mu_{j} \delta_{x_{j}} \text { in } \mathcal{M}(\Omega) \\
\Phi_{*}\left(u_{n}\right) \rightarrow \nu=\Phi_{*}(u)+\sum_{j \in \mathcal{J}} \nu_{j} \delta_{x_{j}} \text { in } \mathcal{M}(\Omega)
\end{array}
$$

and

$$
\nu_{j} \leq \max \left\{S_{N}^{l^{*}} \mu_{j}^{\frac{l^{*}}{l}}, S_{N}^{m^{*}} \mu_{j}^{\frac{m^{*}}{l}}, S_{N}^{l^{*}} \mu_{j}^{\frac{l^{*}}{m}}, S_{N}^{m^{*}} \mu_{j}^{\frac{m^{*}}{m}}\right\}
$$

where $\delta_{x_{j}}$ is the Dirac mass at $x_{j} \in \Omega$ and $S_{N}$ verifies (2.1).

Now, we show that there exists $\alpha_{*}>0$ such that

$$
\Gamma:=\left\{j \in \mathcal{J} ; \nu_{j}>0\right\}=\emptyset \text {, for every } \alpha \in\left(0, \alpha_{*}\right) \text { and } c_{\alpha}<0 .
$$

Let $\psi \in C_{0}^{\infty}\left(\mathbb{R}^{N}\right)$ such that

$$
\psi(x)=1 \text { in } B_{\frac{1}{2}}(0), \operatorname{supp} \psi \subset B_{1}(0) \text { and } 0 \leq \psi(x) \leq 1 \quad \forall x \in \mathbb{R}^{N} .
$$

For each $j \in \Gamma$ and $\epsilon>0$, let us define

$$
\psi_{\epsilon}(x)=\psi\left(\frac{x-x_{j}}{\epsilon}\right), \forall x \in \mathbb{R}^{N} .
$$

Note that $\left(\psi_{\epsilon} u_{n}\right)$ is a bounded sequence in $W_{0}^{1, \Phi}(\Omega)$. Once that

$$
m_{J_{\alpha}}\left(u_{n}\right) \rightarrow 0
$$

by (3.2)

$$
\begin{aligned}
o_{n}(1)= & M\left(\int_{\Omega} \Phi\left(\left|\nabla u_{n}\right|\right) d x\right) \int_{\Omega} \phi\left(\left|\nabla u_{n}\right|\right) \nabla u_{n} \nabla\left(u_{n} \psi_{\epsilon}\right) d x-\alpha \int_{\Omega} h\left(u_{n}\right) u_{n} \psi_{\epsilon} d x \\
& -\left\langle\rho_{n}, \psi_{\epsilon} u_{n}\right\rangle,
\end{aligned}
$$


where $\rho_{n} \in \partial J_{F}\left(u_{n}\right)$.

From (1.1) and Lemma 3.1

$$
\rho_{n} \in\left[\underline{f}\left(x, u_{n}\right), \bar{f}\left(x, u_{n}\right)\right] \text { a.e. } x \in \Omega,
$$

and so, by $\left(f_{1}\right)$

$$
\left|\rho_{n}(x)\right| \leq b_{1} \phi_{*}\left(\left|u_{n}(x)\right|\right)\left|u_{n}(x)\right| \text {, a.e. } x \in \Omega \text {. }
$$

Using the Lemma 2.4 we have that

$$
\left|\int_{\Omega} \rho_{n} u_{n} \psi_{\epsilon} d x\right| \leq b_{1} m^{*} \int_{\Omega} \Phi_{*}\left(u_{n}\right) \psi_{\epsilon} d x .
$$

Once that $\left(u_{n}\right)$ converges strongly for $u$ in $L_{H}(\Omega)$, we have

$$
\int_{\Omega} H\left(u_{n}\right) \psi_{\epsilon} d x \rightarrow \int_{\Omega} H(u) \psi_{\epsilon} d x
$$

From (5.2)-(5.4)

$$
\begin{array}{r}
M\left(\int_{\Omega} \Phi\left(\left|\nabla u_{n}\right|\right) d x\right) \int_{\Omega} \phi\left(\left|\nabla u_{n}\right|\right) \nabla u_{n} \nabla\left(u_{n} \psi_{\epsilon}\right) d x \leq h_{1} \alpha \int_{\Omega} H(u) \psi_{\epsilon} d x \\
+b_{1} m_{*} \int_{\Omega} \Phi_{*}\left(u_{n}\right) \psi_{\epsilon} d x+o_{n}(1) .
\end{array}
$$

On the other hand, by $\left(\phi_{2}\right)$ and $\left(M_{1}\right)$

$$
\begin{aligned}
M\left(\int_{\Omega} \Phi\left(\left|\nabla u_{n}\right|\right) d x\right) & \int_{\Omega} \phi\left(\left|\nabla u_{n}\right|\right) \nabla u_{n} \nabla\left(u_{n} \psi_{\epsilon}\right) d x \\
= & M\left(\int_{\Omega} \Phi\left(\left|\nabla u_{n}\right|\right) d x\right) \int_{\Omega} \phi\left(\left|\nabla u_{n}\right|\right)\left|\nabla u_{n}\right|^{2} \psi_{\epsilon} d x \\
& +M\left(\int_{\Omega} \Phi\left(\left|\nabla u_{n}\right|\right) d x\right) \int_{\Omega} \phi\left(\left|\nabla u_{n}\right|\right)\left(\nabla u_{n} \nabla \psi_{\epsilon}\right) u_{n} d x \\
\geq & l \sigma \int_{\Omega} \Phi\left(\left|\nabla u_{n}\right|\right) \psi_{\epsilon} d x \\
& +M\left(\int_{\Omega} \Phi\left(\left|\nabla u_{n}\right|\right) d x\right) \int_{\Omega} \phi\left(\left|\nabla u_{n}\right|\right)\left(\nabla u_{n} \nabla \psi_{\epsilon}\right) u_{n} d x .
\end{aligned}
$$

By Lemmas 2.2 and 2.6, the sequence

$$
\left\{\left.\left|M\left(\int_{\Omega} \Phi\left(\left|\nabla u_{n}\right|\right) d x\right) \phi\left(\left|\nabla u_{n}\right|\right) \nabla u_{n}\right|\right|_{\widetilde{\Phi}}\right\}
$$

is bounded. Thus, there is a subsequence $\left(u_{n}\right)$ such that

$$
M\left(\int_{\Omega} \Phi\left(\left|\nabla u_{n}\right|\right) d x\right) \phi\left(\left|\nabla u_{n}\right|\right) \nabla u_{n} \rightarrow \widetilde{w}_{1} \text { weakly in } L_{\widetilde{\Phi}}\left(\Omega, \mathbb{R}^{N}\right),
$$


for some $\widetilde{w}_{1} \in L_{\widetilde{\Phi}}\left(\Omega, \mathbb{R}^{N}\right)$. Since $u_{n} \rightarrow u$ in $L_{\Phi}(\Omega)$,

$$
M\left(\int_{\Omega} \Phi\left(\left|\nabla u_{n}\right|\right) d x\right) \int_{\Omega} \phi\left(\left|\nabla u_{n}\right|\right)\left(\nabla u_{n} \nabla \psi_{\epsilon}\right) u_{n} d x \rightarrow \int_{\Omega}\left(\widetilde{w}_{1} \nabla \psi_{\epsilon}\right) u d x .
$$

Thus, combining (5.5), (5.6) and letting $n \rightarrow \infty$, we have

$$
l \sigma \int_{\Omega} \psi_{\epsilon} d \mu+\int_{\Omega}\left(\widetilde{w}_{1} \nabla \psi_{\epsilon}\right) u d x \leq \alpha h_{1} \int_{\Omega} H(u) \psi_{\epsilon} d x+b_{1} m^{*} \int_{\Omega} \psi_{\epsilon} d \nu .
$$

Now we show that

$$
\int_{\Omega}\left(\widetilde{w}_{1} \nabla \psi_{\epsilon}\right) u d x \rightarrow 0, \text { as } \epsilon \rightarrow 0 .
$$

Once that $\left|\left\{\alpha h\left(u_{n}\right) u_{n}+\rho_{n}\right\}\right|_{\widetilde{\Phi}_{*}}$ is bounded, let $\widetilde{w}_{2} \in L_{\widetilde{\Phi}_{*}}(\Omega)$ such that

$$
\alpha h\left(u_{n}\right) u_{n}+\rho_{n} \rightarrow \widetilde{w}_{2} \text { weakly in } L_{\widetilde{\Phi}_{*}}(\Omega) .
$$

Since

$$
M\left(\int_{\Omega} \Phi\left(\left|\nabla u_{n}\right|\right) d x\right) \int_{\Omega} \phi\left(\left|\nabla u_{n}\right|\right) \nabla u_{n} \nabla v d x-\int_{\Omega}\left(\alpha h\left(u_{n}\right) u_{n}+\rho_{n}\right) v d x \rightarrow 0,
$$

as $n \rightarrow+\infty$ for any $v \in W_{0}^{1, \Phi}(\Omega)$,

$$
\int_{\Omega} \widetilde{w}_{1} \nabla v-\widetilde{w}_{2} v d x=0
$$

for any $v \in W_{0}^{1, \Phi}(\Omega)$. Substituting $v=u \psi_{\epsilon}$ we have

$$
\int_{\Omega} \widetilde{w}_{1} \nabla\left(u \psi_{\epsilon}\right)-\widetilde{w}_{2} u \psi_{\epsilon} d x=0 .
$$

Namely,

$$
\int_{\Omega}\left(\widetilde{w}_{1} \nabla \psi_{\epsilon}\right) u d x=-\int_{\Omega}\left(\widetilde{w}_{1} \nabla u-\widetilde{w}_{2} u\right) \psi_{\epsilon} d x
$$

Noting $\widetilde{w}_{1} \nabla u-\widetilde{w}_{2} u \in L^{1}(\Omega)$, we see that right-hand side tends to 0 as $\epsilon \rightarrow 0$. Hence we have

$$
\int_{\Omega}\left(\widetilde{w}_{1} \nabla \psi_{\epsilon}\right) u d x \rightarrow 0
$$

as $\epsilon \rightarrow 0$. Letting $\epsilon \rightarrow 0$ in (5.7), we obtain

$$
l \sigma \mu_{j} \leq b_{1} m^{*} \nu_{j} .
$$

Hence

$$
S_{N}^{-\alpha} \nu_{j} \leq \mu_{j}^{\beta} \leq\left(\frac{b_{1} m^{*}}{l \sigma}\right)^{\beta} \nu_{j}^{\beta},
$$


for some $\alpha \in\left\{l^{*}, m^{*}\right\}$ and $\beta \in\left\{\frac{l^{*}}{l}, \frac{m^{*}}{l}, \frac{l^{*}}{m}, \frac{m^{*}}{m}\right\}$, and so

$$
\nu_{j} \geq\left(\frac{l \sigma}{b_{1} m^{*}}\right)^{\frac{\beta}{\beta-1}} S_{N}^{-\frac{\alpha}{\beta-1}}>0 \text { or } \nu_{j}=0 .
$$

Now, taking $\theta \in\left(2 m, l^{*}\right)$, we get

$$
\begin{aligned}
c_{\alpha}= & \lim _{n \rightarrow+\infty}\left(J_{\alpha}\left(u_{n}\right)-\frac{1}{\theta} J_{\alpha}^{\prime}\left(u_{n}\right) u_{n}\right) \\
& \geq \lim _{n \rightarrow+\infty}\left(\widehat{M}\left(\int_{\Omega} \Phi\left(\left|\nabla u_{n}\right|\right) d x\right)-\frac{m}{\theta} M\left(\int_{\Omega} \Phi\left(\left|\nabla u_{n}\right|\right) d x\right)\right) \int_{\Omega} \Phi\left(\left|\nabla u_{n}\right|\right) d x \\
& -\alpha\left(1-\frac{h_{0}}{\theta}\right) \int_{\Omega} H\left(u_{n}\right) d x-\int_{\Omega}\left(F\left(x, u_{n}\right)-\frac{1}{\theta} \rho_{n} u_{n}\right) d x,
\end{aligned}
$$

and so by $\left(M_{2}\right)$ and $\left(f_{1}\right)$

$$
\begin{array}{r}
c_{\alpha} \geq \lim _{n \rightarrow+\infty}\left(-\alpha\left(1-\frac{h_{0}}{\theta}\right) \int_{\Omega} H\left(u_{n}\right) d x+\left(\frac{b_{0} l^{*}}{\theta}-b_{1}\right) \int_{\Omega} \Phi_{*}\left(u_{n}\right)\right), \\
\quad=-\alpha\left(1-\frac{h_{0}}{\theta}\right) \int_{\Omega} H(u) d x+\left(\frac{b_{0} l^{*}}{\theta}-b_{1}\right)\left(\int_{\Omega} \Phi_{*}(u) d x+\nu_{j}\right),
\end{array}
$$

where $\frac{b_{0} l^{*}}{\theta}-b_{1}>0\left(\right.$ see $\left.\left(f_{1}\right)\right)$. Consequently

$$
\omega_{N}>c_{\alpha} \geq-\alpha\left(1-\frac{h_{0}}{\theta}\right) \eta_{1}\left(|u|_{\Phi_{*}}\right)+\left(\frac{b_{0} l^{*}}{\theta}-b_{1}\right)\left(\xi_{3}\left(|u|_{\Phi_{*}}\right)+\nu_{j}\right) .
$$

Define

$$
\zeta(t)=-\alpha\left(1-\frac{h_{0}}{\theta}\right) \eta_{1}(t)+\left(\frac{b_{0} l^{*}}{\theta}-b_{1}\right) \xi_{3}(t), t \geq 0,
$$

note that

$$
\zeta(t)=\left\{\begin{array}{l}
-\alpha\left(1-\frac{h_{0}}{\theta}\right) t^{h_{0}}+\left(\frac{b_{0} l^{*}}{\theta}-b_{1}\right) t^{m^{*}}, \quad \text { se } t \in(0,1] \\
-\alpha\left(1-\frac{h_{0}}{\theta}\right) t^{h_{1}}+\left(\frac{b_{0} l^{*}}{\theta}-b_{1}\right) t^{t^{*}}, \quad \text { se } t \in[1,+\infty) .
\end{array}\right.
$$

This function attains its absolute minimum, for $t>0$, at the point

$$
t_{0}:=\left(\frac{\alpha\left(\theta-h_{0}\right) h_{0}}{\left(b_{0} l^{*}-b_{1} \theta\right) m^{*}}\right)^{\frac{h_{0}-1}{m^{*}-1}} .
$$

Thus, we conclude for $\alpha>0$ small enough that

$$
\begin{aligned}
& \omega_{N}+\alpha^{h_{0}\left(\frac{h_{0}-1}{m^{*}-1}\right)+1}\left(1-\frac{h_{0}}{\theta}\right) \lambda_{0}^{h_{0}}-\alpha^{m^{*}\left(\frac{b_{0}-1}{m^{*}-1}\right)}\left(\frac{b_{0} l^{*}}{\theta}-b_{1}\right) \lambda_{0}^{m^{*}}>\nu_{j}, \\
& \text { where } \lambda_{0}:=\left(\frac{\left(\theta-h_{0}\right) h_{0}}{\left(b_{0} l^{*}-b_{1} \theta\right) m^{*}}\right)^{\frac{h_{0}-1}{m^{*}-1}}>0 .
\end{aligned}
$$


Supposing that there exists $j \in \Gamma$ such that $\nu_{j}>0$. From (5.8) and (5.10)

$$
\left(1-\frac{h_{0}}{\theta}\right) \lambda_{0}^{h_{0}} \alpha^{h_{0}\left(\frac{h_{0}-1}{m^{*}-1}\right)+1}-\left(\frac{b_{0} l^{*}}{\theta}-b_{1}\right) \lambda_{0}^{m^{*}} \alpha^{m^{*}\left(\frac{h_{0}-1}{m^{*}-1}\right)}>\frac{1}{2} \omega_{N},
$$

but this is a contradiction, for $\alpha>0$ small enough. Showing that

$$
u_{n} \rightarrow u \text { in } L_{\Phi_{*}}(\Omega) .
$$

Lemma 5.2 Let $\left(u_{n}\right) \subset W_{0}^{1, \Phi}(\Omega)$ the $(P S)_{c_{\alpha}}$ sequence obtained in the previous. Then, for some subsequence, still denoted by it self,

$$
u_{n} \rightarrow u \text { in } W_{0}^{1, \Phi}(\Omega) \text {. }
$$

Proof: Now, as $m_{J_{\alpha}}\left(u_{n}\right)=o_{n}(1)$, the last limit gives

$M\left(\int_{\Omega} \Phi\left(\left|\nabla u_{n}\right|\right) d x\right) \int_{\Omega} \phi\left(\left|\nabla u_{n}\right|\right)\left|u_{n}\right|^{2} d x=\alpha \int_{\Omega} h\left(u_{n}\right) u_{n}^{2} d x+\int_{\Omega} \rho_{n} u_{n} d x+o_{n}(1)$.

In what follows, let us denote by $\left\{P_{n}\right\}$ the following sequence,

$$
P_{n}(x)=\left\langle\phi\left(\left|\nabla u_{n}(x)\right|\right) \nabla u_{n}(x)-\phi(|\nabla u(x)|) \nabla u(x), \nabla u_{n}(x)-\nabla u(x)\right\rangle .
$$

Since $\Phi$ is convex in $\mathbb{R}$ and $\Phi(||$.$) is C^{1}$ class in $\mathbb{R}^{N}$, has $P_{n}(x) \geq 0$. From definition of $\left\{P_{n}\right\}$,

$$
\int_{\Omega} P_{n} d x=\int_{\Omega} \phi\left(\left|\nabla u_{n}\right|\right)\left|\nabla u_{n}\right|^{2} d x-\int_{\Omega} \phi\left(\left|\nabla u_{n}\right|\right) \nabla u_{n} \nabla u d x-\int_{\Omega} \phi(|\nabla u|) \nabla u \nabla\left(u_{n}-u\right) d x .
$$

Recalling that $u_{n} \rightarrow u$ in $W_{0}^{1, \Phi}(\Omega)$, we have

$$
\int_{\Omega} \phi(|\nabla u|) \nabla u \nabla\left(u_{n}-u\right) d x \rightarrow 0 \quad \text { as } n \rightarrow \infty
$$

which implies that

$$
\int_{\Omega} P_{n} d x=\int_{\Omega} \phi\left(\left|\nabla u_{n}\right|\right)\left|\nabla u_{n}\right|^{2} d x-\int_{\Omega} \phi\left(\left|\nabla u_{n}\right|\right) \nabla u_{n} \nabla u d x+o_{n}(1) .
$$

On the other hand

$$
\begin{aligned}
0 \leq M\left(\int_{\Omega} \Phi\left(\left|\nabla u_{n}\right|\right) d x\right) \int_{\Omega} P_{n} d x= & \alpha \int_{\Omega} h\left(u_{n}\right) u_{n}^{2} d x-\alpha \int_{\Omega} h\left(u_{n}\right) u_{n} u d x \\
& +\int_{\Omega} \rho_{n} u_{n} d x-\int_{\Omega} \rho_{n} u d x+o_{n}(1),
\end{aligned}
$$

where $\rho_{n} \in \partial J_{F}\left(u_{n}\right)$. Once that $\left|\rho_{n}\right|_{\widetilde{\Phi}_{*}}$ is bounded, we have that

$$
\begin{aligned}
\int_{\Omega} P_{n} d x & \leq \frac{\alpha}{\sigma} \int_{\Omega} h\left(u_{n}\right) u_{n}\left(u_{n}-u\right) d x+\frac{1}{\sigma} \int_{\Omega} \rho_{n}\left(u_{n}-u\right) d x \\
& \leq \frac{\alpha}{\sigma}\left|u_{n}\right|_{\widetilde{H}}\left|u_{n}-u\right|_{H}+\frac{1}{\sigma}\left|\rho_{n}\right|_{\widetilde{\Phi}_{*}}\left|u_{n}-u\right|_{\Phi_{*}} \rightarrow 0 .
\end{aligned}
$$


Applying a result due to Dal Maso and Murat 25]

$$
u_{n} \rightarrow u \text { in } W_{0}^{1, \Phi}(\Omega) \text {. }
$$

Lemma 5.3 If $I_{\alpha}(u)<0$, then $\int_{\Omega} \Phi(|\nabla u|) d x<A_{\alpha}$ and $J_{\alpha}(v)=I_{\alpha}(v)$, for all $v$ in a sufficiently small neighborhood of $u$. Moreover, $I_{\alpha}$ verifies a local Palais-Smale condition for $c_{\alpha}<0$.

Proof: Since $\bar{g}\left(\int_{\Omega} \Phi(|\nabla u|) d x\right) \leq I_{\alpha}(u)<0$, we have that $\int_{\Omega} \Phi(|\nabla u|) d x<A_{\alpha}$. Once that $\left\{v \in W_{0}^{1, \Phi}(\Omega) ; \int_{\Omega} \Phi(|\nabla v|) d x<A_{\alpha}\right\}$ is open, there exists $r_{\alpha}>0$ such that $\int_{\Omega} \Phi(|\nabla v|) d x<A_{\alpha}$ for all $v \in B_{r_{\alpha}}(u)$ arguing as in Theorem 1.1 we conclude that $J_{\alpha}(v)=I_{\alpha}(v)$, for all $v \in B_{r_{\alpha}}(u)$. Moreover, if $\left(u_{n}\right)$ is a sequence such that $I_{\alpha}\left(u_{n}\right) \rightarrow c_{\alpha}<0$ and $m_{I_{\alpha}}\left(u_{n}\right) \rightarrow 0$, then, for $n$ sufficiently large, $J_{\alpha}\left(u_{n}\right)=I_{\alpha}\left(u_{n}\right) \rightarrow c_{\alpha}<0$ and $m_{J_{\alpha}}\left(u_{n}\right)=m_{I_{\alpha}}\left(u_{n}\right) \rightarrow 0$. Since $I_{\alpha}$ is coercive, we get that $\left(u_{n}\right)$ is bounded in $W_{0}^{1, \Phi}(\Omega)$. From Lemma 5.2 for $\alpha$ sufficiently small and $c_{\alpha}<0$, hence, up to a subsequence, $\left(u_{n}\right)$ is strongly convergent in $W_{0}^{1, \Phi}(\Omega)$.

Let $K_{c}$ be the set of critical points of $J$. More precisely

$$
K_{c}(J)=\left\{u \in W_{0}^{1} L_{\Phi}(\Omega): 0 \in \partial J(u) \text { and } J(u)=c\right\} .
$$

Since $I_{\alpha}$ is even, we have that $K_{c_{\alpha}}:=K_{c_{\alpha}}\left(I_{\alpha}\right)$ is symmetric. The next result is important in our arguments and allows we conclude that $K_{c_{\alpha}}$ is compact. The proof can be found in 14.

Lemma 5.4 If $I_{\alpha}$ satisfies the nonsmooth $(P S)_{c_{\alpha}}$ condition, then $K_{c_{\alpha}}$ is compact.

To prove that $K_{c_{\alpha}}$ does not contain zero, we construct a special class of the levels $c_{\alpha}$.

For each $k \in \mathbb{N}$, we define the set

$$
\Gamma_{k}=\left\{C \subset W_{0}^{1} L_{\Phi}(\Omega): C \text { is closed, } C=-C \text { and } \gamma(C) \geq k\right\},
$$

and the values

$$
c_{k}^{\alpha}=\inf _{C \in \Gamma_{k}} \sup _{u \in C} J_{\alpha}(u)
$$

Note that

$$
-\infty \leq c_{1}^{\alpha} \leq c_{2}^{\alpha} \leq c_{3}^{\alpha} \leq \ldots \leq c_{k}^{\alpha} \leq \cdots
$$

and, once that $I_{\alpha}$ is coercive and continuous, $I_{\alpha}$ is bounded below and, hence, $c_{1}^{\alpha}>-\infty$. In this case, arguing as in [9, Proposition 3.1], we can prove that each $c_{k}^{\alpha}$ is a critical value for the functional $I_{\alpha}$.

Lemma 5.5 Given $k \in \mathbb{N}$, there exists $\epsilon=\epsilon(k)>0$ such that

$$
\gamma\left(I_{\alpha}^{-\epsilon}\right) \geq k,
$$

where $I_{\alpha}^{-\epsilon}=\left\{u \in W_{0}^{1} L_{\Phi}(\Omega): I_{\alpha}(u) \leq-\epsilon\right\}$. 
Proof: Fix $k \in \mathbb{N}$, let $X_{k}$ be a k-dimensional subspace of $W_{0}^{1} L_{\Phi}(\Omega)$. Thus, there exists $C_{k}>0$ such that

$$
-C_{k}\|u\|_{\Phi} \geq-|u|_{H}
$$

for all $u \in X_{k}$.

Thus, for all $R \in(0,1)$ and for all $u \in X_{k}$ with $\int_{\Omega} \Phi(|\nabla u|) d x<R$, from continuity of the function $M$, we conclude that, there exists $C$, such that

$$
\begin{aligned}
I_{\alpha}(u) & \leq C \xi_{1}\left(\|u\|_{\Phi}\right)-\alpha \eta_{0}\left(C_{k}\|u\|_{\Phi}\right) \\
& =C\|u\|_{\Phi}^{l}-\alpha C_{k}^{h_{1}}\|u\|_{\Phi}^{h_{1}} .
\end{aligned}
$$

Fixing $r \in(0,1)$ such that

$$
C r^{l-h_{1}}<\alpha C_{k}^{h_{1}},
$$

and $\mathcal{S}_{r}=\left\{u \in X_{k} ;\|u\|_{\Phi}=r\right\}$, we get

$$
I_{\alpha}(u) \leq C r^{l}-\alpha C_{k} r^{h_{1}}<0=I_{\alpha}(0), \forall u \in \mathcal{S}_{r},
$$

which implies there exists $\epsilon=\epsilon(r)>0$ such that

$$
I_{\alpha}(u)<-\epsilon<0,
$$

for all $u \in \mathcal{S}_{r}$. Since $X_{k}$ and $\mathbb{R}^{k}$ are isomorphic and $\mathcal{S}_{r}$ and $S^{k-1}$ are homeomorphic, we conclude from Corollary 4.1 that $\gamma\left(\mathcal{S}_{r}\right)=\gamma\left(S^{k-1}\right)=k$. Moreover, once that $\mathcal{S}_{r} \subset I_{\alpha}^{-\epsilon}$ and $I_{\alpha}^{-\epsilon}$ is symmetric and closed, we have

$$
k=\gamma\left(\mathcal{S}_{r}\right) \leq \gamma\left(I_{\alpha}^{-\epsilon}\right) .
$$

Lemma 5.6 Given $k \in \mathbb{N}$, the number $c_{k}^{\alpha}$ is negative.

Proof: From Lemma 5.5, for each $k \in \mathbb{N}$ there exists $\epsilon>0$ such that $\gamma\left(I_{\alpha}^{-\epsilon}\right) \geq k$. Moreover, $0 \notin I_{\alpha}^{-\epsilon}$ and $I_{\alpha}^{-\epsilon} \in \Gamma_{k}$. On the other hand

$$
\sup _{u \in I_{\alpha}^{-\epsilon}} I_{\alpha}(u) \leq-\epsilon
$$

Hence,

$$
-\infty<c_{k}^{\alpha}=\inf _{C \in \Gamma_{k}} \sup _{u \in C} I_{\alpha}(u) \leq \sup _{u \in I_{\alpha}^{-\epsilon}} I_{\alpha}(u) \leq-\epsilon<0 .
$$

The next result is a direct adaptation of [8, Lemma 4.4]. See also 28, Lemma 7.5].

Lemma 5.7 If $c_{k}^{\alpha}=c_{k+1}^{\alpha}=\ldots=c_{k+r}^{\alpha}$ for some $r \in \mathbb{N}$, then

$$
\gamma\left(K_{c_{k}^{\alpha}}\right) \geq r+1 \text {. }
$$




\subsection{Proof of Theorem 1.1}

If $-\infty<c_{1}^{\alpha}<c_{2}^{\alpha}<\ldots<c_{k}^{\alpha}<\ldots<0$ and since each $c_{k}^{\alpha}$ critical value of $I^{\alpha}=J^{\alpha}$ (see Lemma 5.3), then we obtain infinitely many critical points of $J_{\alpha}$.

On the other hand, if there are two constants $c_{k}^{\alpha}=c_{k+r}^{\alpha}$, then $c_{k}^{\alpha}=c_{k+1}^{\alpha}=$ $\ldots=c_{k+r}^{\alpha}$ and from Lemma 5.7, we have

$$
\gamma\left(K_{c_{k}^{\alpha}}\right) \geq r+1 \geq 2 .
$$

From Proposition 4.2, $K_{c_{k}^{\alpha}}$ has infinitely many points.

Let $\left(u_{k}\right)$ critical points of $J_{\alpha}$. Now we show that, for

$$
a_{0}<\eta_{1}^{-1}\left(\frac{\sigma l}{H(1)|\Omega| \alpha h_{1}} \xi_{0}(C)\right)
$$

we have that

$$
\left\{x \in \Omega:\left|u_{k}(x)\right| \geq a_{0}\right\}
$$

has positive measure. Thus every critical points of $J_{\alpha}$, are solutions of $(P)$. Suppose, by contradiction, that this set has null measure. Thus

$$
\begin{aligned}
0 & =M\left(\int_{\Omega} \Phi\left(\left|\nabla u_{k}\right|\right) d x\right) \int_{\Omega} \phi\left(\left|\nabla u_{k}\right|\right)\left|\nabla u_{k}\right|^{2} d x-\alpha \int_{\Omega} h\left(u_{k}\right) d x \\
& \geq \sigma l \int_{\Omega} \Phi\left(\left|\nabla u_{k}\right|\right) d x-\alpha h_{1} \int_{\Omega} H\left(u_{k}\right) d x \\
& \geq \sigma l \xi_{0}\left(\left\|u_{k}\right\|_{\Phi}\right)-\alpha h_{1} H\left(a_{0}\right)|\Omega|,
\end{aligned}
$$

where we conclude

$$
\sigma l \xi_{0}\left(\left\|u_{k}\right\|_{\Phi}\right)^{(\alpha+1)} \leq \alpha h_{1} \eta_{1}\left(a_{0}\right)|\Omega| H(1) .
$$

Since $c_{k}^{\alpha} \leq-\epsilon<0$, there exists $C>0$ such that $\left\|u_{k}\right\| \geq C>0$. Hence

$$
a_{0} \geq \eta_{1}^{-1}\left(\frac{\sigma l}{H(1)|\Omega| \alpha h_{1}} \xi_{0}(C)\right)
$$

which contradicts (5.12). Then,

$$
\left\{x \in \Omega:\left|u_{k}(x)\right| \geq a_{0}\right\}
$$

has positive measure.

\section{References}

[1] A. Adams and J. F. Fournier, Sobolev spaces, 2nd ed., Academic Press, (2003).

[2] C.O. Alves, F.J.S.A. Corrêa and T.F Ma, Positive solutions for a quasilinear elliptic equation of Kirchhoff type, Comput. Math. Appl., 49(2005)85-93. 
[3] C.O. Alves and F.J.S.A. Corrêa, On existence of solutions for a class of problem involving a nonlinear operator, Comm. Appl. Nonlinear Anal., 8(2001)43-56.

[4] C.O. Alves, J.V. Gonçalves and J.A. Santos, Strongly Nonlinear Multivalued Elliptic Equations on a Bounded Domain, J Glob Optim, DOI 10.1007/s10898-013-0052-3, 2013.

[5] A. Ambrosetti and P. H Rabinowitz, Dual variational methods in critical point theory and apllications, J. Functional Analysis, vol 14(1973)349-381.

[6] A. Arosio, On the nonlinear Timoshenko-Kirchoff beam equation, Chin. Annal Math., 20 (1999), 495-506.

[7] A. Arosio, A geometrical nonlinear correction to the Timoshenko beam equation, Nonlinear Anal. 47(2001), 729-740.

[8] J. G. Azorero and I. P. Alonso, Multiplicity of solutions for elliptic problems with critical exponent or with a nonsymmetric term, Trans. Amer. Math. Soc., vol 323 n. 2(1991)877-895.

[9] T. Bartsch, T. Weth and M. Willem, Partial symmetry of least energy nodal solution to some variational problems, J. d'Analyse Mathematique, 96(2005), 1-18.

[10] G. Bonanno, G. M. Bisci and V. Radulescu, Quasilinear elliptic nonhomogeneous Dirichlet problems through OrliczSobolev spaces, Nonlinear Analysis 75 (2012) 4441-4456.

[11] H. Brezis. and E. Lieb, A relation between pointwise convergence of functions and convergence of functinals, Proc. Amer. Math. Soc. 88 (1983), 486-490.

[12] A. Castro, Metodos variacionales y analisi functional no linear, X Coloquio colombiano de Matematicas, 1980.

[13] M.L. Carvalho and J.V. Goncalves, Multivalued Equations on a Bounded Domain via Minimization on Orlicz-Sobolev, Journal of Convex Analysis, preprint 2014.

[14] K.C. Chang, Variational methods for nondifferentiable functionals and their applications to partial differential equations, J. Math. Anal., 80 (1981)102129.

[15] K. C. Chang, On the multiple solutions of the elliptic differential equations with discontinuous nonlinear terms Sci. Sinica 21 (1978) 139-158.

[16] K. C. Chang, The obstacle problem and partial differential equations with discontinuous nonlinearities Comm. Pure Appl. Math (1978) 139-158. 
[17] N. T. Chung and H. Q. Toan, On a nonlinear and non-homogeneous problem without $(A-R)$ type condition in OrliczSobolev spaces, Appl. Math. and Comput. 219 (2013) 7820-7829.

[18] N. T. Chung, Multiple solutions for a nonlocal problem in Orlicz-Sobolev spaces, Ricerche mat (2013), DOI 10.1007/s11587-013-0171-7.

[19] D.C. Clark, A variant of the Lusternik-Schnirelman theory, Indiana Univ. Math. J., 22(1972)65-74.

[20] F.H. Clarke,Optimization and Nonsmooth Analysis, John Wiley \& Sons, N.Y, 1983.

[21] F.H. Clarke, Generalized gradients and applications, Trans. Amer. Math. Soc. 265 (1975), 247-262.

[22] F.J. S. A. Corrêa and G. M. Figueiredo, On a p-Kirchhoff equation via Krasnoselskii's genus, Applied Math. Letters, 22(2009)819-822.

[23] F.J. S. A. Corrêa and R. G. Nascimento, Existence of solutions to nonlocal elliptic equations with discontinuous terms, EJDE 26(2012) 1-14.

[24] D. G. Costa An invitation to variational methods in Differential Equations, Birkhaüser Boston, 2007.

[25] G. Dal Maso and F. Murat, Almost everywhere convergence of gradients of solutions to nonlinear elliptic systems, Nonlinear Anal. 31 (1998), 405-412.

[26] M. Mihailescu and D. Repovs, Multiple solutions for a nonlinear and nonhomogeneous problem in Orlicz-Sobolev spaces, Appl. Math. and Comput. 217 (2011) 6624-6632.

[27] T.K. Donaldson and N.S. Trudinger, Orlicz-Sobolev spaces and imbedding theorems, J. Funct. Anal. 8 (1971) 52-75.

[28] G. M. Figueiredo and J.R. dos Santos Junior, Multiplicity of solutions for a Kirchhoff equation with subcritical or critical growth, DIE-Diff. Int. Equations, 25(2012), 853-868.

[29] N. Fukagai and K. Narukawa, Positive solutons of quasilinear elliptic equations with critical Orlicz-Sobolev nonlinearity on $\mathbb{R}^{N}$, Funkciallaj Ekvacioj, 49(2006)235-267.

[30] M. R. Grossinho and S. A. Tersian An Introduction to Minimax theorems and their Applications to Differential Equations, 2001.

[31] J.P. Gossez Orlicz-Sobolev spaces and nonlinear elliptic boundary value problems. In: Fuk, Svatopluk and Kufner, Alois (eds.): Nonlinear Analysis, Function Spaces and Applications, Proceedings of a Spring School held in Horn Bradlo, 1978. [Vol 1]. BSB B. G. Teubner Verlagsgesellschaft, Leipzig, 1979. pp. 59-94. 
[32] X. He and W. Zou, Multiplicity of Solutions for a Class of Kirchhoff Type Problems, Acta Math. Applicatae Sinica, 26(2010), 387-394.

[33] M. A. Kranolseskii, Topological methods in the theory of nonlinear integral equations. MacMillan, New York, 1964.

[34] G. Kirchhoff Mechanik, Teubner,Leipzig, 1883.

[35] J.L. Lions On some questions in boundary value problems of mathematical physics International Symposium on Continuum, Mechanics and Partial Differential Equations, Rio de Janeiro(1977), Mathematics Studies, Vol. 30, North-Holland, Amsterdam (1978)284-346.

[36] T. F. Ma, Remarks on an elliptic equation of Kirchhoff type. Nonlinear Anal., 63(2005,)1967-1977.

[37] J. Sun and C. Tang, Existence and multiplicity of solutions for Kirchhoff type equations, Nonlinear Anal., 74 (2011), 1212-1222.

[38] J. A. Santos, Multiplicity of solutions for quasilinear equations involving critical Orlicz-Sobolev nonlinear terms, EJDE, Vol. 2013 (2013), No. 249, pp. 1-13. 